\title{
Leukocyte depletion does not affect the in vitro healing ability of platelet rich plasma
}

\author{
ILARIA GIUSTI $^{1}$, MARIANNA DI FRANCESCO ${ }^{1}$, SANDRA D'ASCENZO $^{1}$, \\ PAOLA PALUMBO ${ }^{1}$, ANNA RUGHETTI ${ }^{2}$, LUIGI DELL'ORSO ${ }^{2}$, PAOLA ANNUNZIATA VARASANO $^{3}$, \\ GABRIELLA LUCIDI PRESSANTI ${ }^{3}$ and VINCENZA DOLO ${ }^{1}$ \\ ${ }^{1}$ Department of Life, Health and Environmental Sciences, University of L'Aquila; \\ ${ }^{2}$ Immunotransfusion Medicine Unit, 'San Salvatore' Hospital, I-67100 L'Aquila; \\ ${ }^{3}$ Immunotransfusion Medicine Unit, 'Giuseppe Mazzini’ Hospital, I-64100 Teramo, Italy
}

Received July 19, 2017; Accepted January 19, 2018

DOI: $10.3892 /$ etm.2018.5887

\begin{abstract}
The clinical use of platelet-rich plasma (PRP) containing or deprived of leukocytes remains a subject of debate and a controversial issue. It is not yet clear whether leukocyte content has a positive or negative effect on tissue healing processes. Several studies, conducted mainly in the orthopedic field, support the use of leukocyte-poor (LP) PRP, whereas other studies have not identified any significant differences between the use of LP and leukocyte-rich PRP. In the present study, the role of leukocytes contained in PRP was assessed to verify their in vitro effect on fibroblasts and endothelial cells, which have a leading role in the biological processes associated with wound healing (including angiogenesis and matrix remodeling). The original sample of PRP was divided into two aliquots, one of which remained unaltered, while the other was deprived of leukocytes. The two aliquots were used in in vitro tests in order to verify the effects of leukocytes on proliferation, wound healing and tube formation, and in molecular analyses of growth factor and enzyme content. The present results highlighted a substantial overlap between the two formulations. This may be explained by similar levels of growth factors (vascular endothelial growth factor, thrombospondin-1, interferon- $\gamma$, platelet-derived growth factor-BB, -AA and -B, tumor growth factor- $\beta 1$, fibroblast growth factor 7 and tumor necrosis factor- $\alpha$ ) and enzymes (gelatinases and plasminogen activators) in the two formulations. These results support the hypothesis that the ability of the PRP to affect the in vitro biological response of endothelial cells and fibroblasts does not rely on the presence of leukocytes.
\end{abstract}

Correspondence to: Professor Vincenza Dolo, Department of Life, Health and Environmental Sciences, University of L'Aquila, Via Vetoio-Coppito II, I-67100 L'Aquila, Italy

E-mail: vincenza.dolo@univaq.it

Key words: platelet-rich plasma, wound healing, endothelial cells, fibroblasts, leukocyte depletion

\section{Introduction}

In recent years, the use of blood components for purposes other than classic transfusion has become more popular. It has emerged that blood cells and/or plasma-derived factors may have therapeutic effects in various diseases. There is increasing interest in the use of platelet derivatives, particularly platelet-rich plasma (PRP), which is classically described as 'a volume of plasma that has a platelet count above baseline (of whole blood)' (1). Platelets are not only involved in a well-documented haemostatic function, but also play a fundamental role in tissue repair and regeneration. They have the ability to store numerous growth factors (GFs), enzymes and other bioactive molecules, which are rapidly released following platelet activation (2-4). Platelets are also able to stimulate certain key events in the reparative processes, such as replication of cells of mesenchymal origin (including fibroblasts, osteoblasts and endothelial cells), or chemotactic effects (2-5).

Various methods have been developed for the topical use of platelet concentrates (PCs) in order to stimulate tissue growth and regeneration. Their clinical use in wound regeneration has gradually expanded over recent years, being applied in various fields such as ophthalmology, pain management, oral and maxillofacial surgery, orthopedics, plastic, periodontal and cardiac surgery and sports medicine (5-11).

PRP contributes to tissue healing since it is a carrier of all the individual elements involved in this process (including platelets, leukocytes and GFs). Establishing the role and importance of each of these factors is essential in order to identify the most effective product to induce tissue healing.

The purpose of the present study was to evaluate to what extent the presence of leukocytes in PRP influences its ability to induce, in vitro, the biological activities that are necessary for wound repair in cell types that are involved in the tissue regeneration process. These cell types included endothelial cells (required for angiogenesis) and fibroblasts (required for matrix remodeling). 


\section{Materials and methods}

Platelet gel $(P G)$-released supernatant preparation. Whole blood samples (each $450 \mathrm{ml}$ ) were collected by standard homologous blood donation using triple bags (Teruflex with CPD; S.A.G.M., Terumo, Rome, Italy). Each donor provided consent according to current laws (Decree Law 3, March 2005, and Law 21, October 2005, n. 219).

PRP was produced from the blood donation of each patient. Fractionation was carried out by initial centrifugation of the bag for $10 \mathrm{~min}$ at $22^{\circ} \mathrm{C}$ at $462 \mathrm{x} \mathrm{g}$ using a Heraeus Cryofuge 6000i centrifuge [AHSI SpA, Massa Martana (PG), Italy] to obtain PRP and red cell concentrates. Subsequently, the obtained PRP was subjected to a second centrifugation for $6 \mathrm{~min}$ at $22^{\circ} \mathrm{C}$ at $3,932 \mathrm{x}$ g to produce the PC and platelet-poor plasma. Finally, the platelets were hyperconcentrated in $10-15 \mathrm{ml}$ of plasma.

This process produced leukocyte-rich PC (LR-PC), which was later divided into two aliquots. One aliquot was deprived of leukocytes through filtration on TERUFLEX BP-KIT ${ }^{\circledR}$ filters (Terumo, Rome, Italy) to obtain leukocyte-poor PC (LP-PC).

In order to measure platelet content and, when necessary, the depletion of leukocytes, both whole blood and obtained PCs were analyzed using a Coulter ${ }^{\circledR} \mathrm{A}^{\mathrm{c}} \cdot \mathrm{T}$ 5diff AL hemocytometer (Beckman Coulter, Inc., Brea, CA, USA).

LR-PG or LP-PG was produced by placing LR-PC or LP-PC, respectively, in Vacutainer Plus tubes (BD Biosciences, Plymouth, UK) containing $5 \mathrm{NIH}$ units thrombin. Calcium gluconate [Bioindustria Laboratorio Italiano Medicinali SpA, Novi Ligure (AL), Italy] was added at a 1:20 dilution. Subsequently, the solutions were allowed to clot for 5-10 min at $37^{\circ} \mathrm{C}$. After a further $20-25 \mathrm{~min}$, the clots were fully retracted and were centrifuged for $10 \mathrm{~min}$ at $153 \mathrm{x}$ g to obtain a supernatant that was rich in GFs released from the activated platelets and, where present, leukocytes. The supernatants were subjected to a succession of centrifugations to remove red cells, debris and cellular stroma, and were immediately used in experiments. For convenience, LR-PG- and LP-PG-derived supernatants will be identified hereafter as 'LR-PGs' and 'LP-PGs'.

Cell culture. Human umbilical vein endothelial cells (HUVECs) were isolated from umbilical cord veins. The cells were grown at $37^{\circ} \mathrm{C}$ and $5 \% \mathrm{CO}_{2}$ on $1 \%$ gelatin-coated flasks in DMEM supplemented with $10 \%$ fetal bovine serum (FBS), $10 \%$ newborn calf serum (NCS), $20 \mathrm{mM} \mathrm{N}$-(2-hydroxyethyl) piperazine-N'-(2-ethanesulfonic acid) (HEPES), $6 \mathrm{U} / \mathrm{ml}$ heparin, $2 \mathrm{mM}$ glutamine, $50 \mu \mathrm{g} / \mathrm{ml}$ endothelial cell growth factor (ECGF), penicillin and streptomycin. The cells were used between the third and fifth passage.

Normal human dermal fibroblasts (NHDFs) were purchased from Lonza (Walkersville, MD, USA) and grown at $37^{\circ} \mathrm{C}$ and $5 \% \mathrm{CO}_{2}$ in DMEM supplemented with $10 \% \mathrm{FBS}$, $2 \mathrm{mM}$ glutamine, penicillin and streptomycin. The cells were used before reaching their 15th doubling, as recommended by the supplier.

HEPES and ECGF were purchased from Sigma-Aldrich (Merck KGaA, Darmstadt, Germany). FBS, DMEM, glutamine, penicillin and streptomycin were purchased from Euroclone SpA (Milan, Italy). NCS was purchased from Gibco (Thermo Fisher Scientific, Inc., Waltham, MA, USA).
Cell proliferation. Cell proliferation was determined using an XTT assay (Sigma-Aldrich; Merck KGaA). Living cells metabolically reduce XTT mixed with phenazine methosulfate (Sigma-Aldrich; Merck KGaA) to produce a colored, non-toxic, water-soluble formazan; its optical density is directly proportional to the number of viable cells.

Briefly, 1,250 HUVECs/well were seeded into a $1 \%$ gelatin-coated 96-well plate, then treated with LR-PGs and LP-PGs $\left(0-2.5 \times 10^{6}\right.$ platelets $\left./ \mu \mathrm{l}\right)$, diluting the original preparation with DMEM supplemented with HEPES, heparin, ECGF and $2 \% \mathrm{FBS}$; the same medium was used for the control (untreated cells). NHDFs were seeded at 1,000 cells/well into a 96-well plate and then treated with LR-PGs or LP-PGs $\left(0-3 \times 10^{6}\right.$ platelets $\left./ \mu 1\right)$, diluting the original preparation with DMEM supplemented with $1 \%$ FBS; the same medium was used for the control (untreated cells).

After seeding, HUVECs and NHDFs were incubated for 24 or $48 \mathrm{~h}$, respectively, in complete medium at $37^{\circ} \mathrm{C}$ and $5 \% \mathrm{CO}_{2}$ to enable cell adhesion and spreading prior to $\mathrm{PG}$ treatments. Once treated, cells were incubated at $37^{\circ} \mathrm{C}$ with $5 \% \mathrm{CO}_{2}$ for $72 \mathrm{~h}$. At the end of this period, an XTT assay was performed and the optical density was evaluated at $450 \mathrm{~nm}$. XTT tests were performed before the cells reached confluence to prevent possible artifact decreases in the results due to contact inhibition.

Each experiment was performed in triplicate and repeated at least twice. Data are expressed as the mean \pm standard deviation.

Tube formation assay. A tube formation assay was performed to measure the ability of endothelial cells to invade, migrate, organize, and differentiate into capillary-like tubular structures. This was assessed within a three-dimensional matrix constituted by $10 \mathrm{mg} / \mathrm{ml}$ Matrigel Growth Factor Reduced (BD Biosciences, Franklin Lakes, NJ, USA). HUVECs (20,000 cells/well) were seeded onto Matrigel-coated 96-well plates in DMEM containing 1\% FBS or in LR-PGs or LP-PGs diluted in the same medium to reach the desired concentration. After $6 \mathrm{~h}$, the formation of tubes was photographed using an inverted optical microscope (Zeiss, Oberkochen, Germany) supported by a Nikon camera (Nikon, Tokyo, Japan). The images were independently scored by two blinded observers. Several images were acquired per well and processed using the Angiogenesis Analyzer plugin on ImageJ software.

In vitro wound-healing assay. The wound-healing assay is one of the earliest developed tests to study directional cell migration in vitro. It is based on the observation of cell migration into a scratch 'wound' created on a cell monolayer.

HUVECs and NHDFs were cultured in 24-well microplates under normal culture conditions and allowed to reach maximum confluence. A previously sterilized round-tipped steel needle was used to create several scratch wounds of approximately $0.2 \mathrm{~mm}$ in the cellular stratum. The microplates were washed three times with DMEM and the cells were cultured in a low $1 \%$ FBS medium (untreated cells) or with LR-PGs and LP-PGs diluted to the desired concentrations $\left(0.3 \times 10^{6}, 1.5 \times 10^{6}\right.$ and $2.5 \times 10^{6}$ platelets $\left./ \mathrm{ml}\right)$ with the same medium. The status of the scratch wounds was controlled using contrast-phase microscopy at the beginning of the assay 
and at regular intervals. Representative images were obtained (after $6 \mathrm{~h}$ for HUVECs and $24 \mathrm{~h}$ for NHDFs) using a Nikon camera (Nikon, Tokyo, Japan). Where possible, the percentage of closed area relative to the original wound area was measured using ImageJ software.

Zymography. Gelatin zymography was performed using sodium dodecyl sulfate-polyacrylamide gels (SDS-PAGE; 7.5\%) co-polymerized with $1 \mathrm{mg} / \mathrm{ml}$ gelatin type B (Sigma-Aldrich; Merck KGaA). Casein-plasminogen zymography was performed using $10 \%$ SDS-PAGE co-polymerized with $0.2 \%$ casein and $10 \mathrm{mg} / \mathrm{ml}$ plasminogen (both from Sigma-Aldrich; Merck KGaA). Zymographies were performed on supernatants diluted in SDS-PAGE sample buffer in non-reducing conditions without heating; volumes corresponding to $5 \times 10^{6}$ and $20 \times 10^{6}$ platelets (for gelatin and casein-plasminogen zymography, respectively) were loaded for both LR-PGs and LP-PGs. After electrophoresis, the gels were washed twice for $30 \mathrm{~min}$ in $50 \mathrm{mM}$ Tris ( $\mathrm{pH} 7.4$ ) with $2.5 \%$ Triton $\mathrm{X}-100$ at room temperature and incubated overnight at $37^{\circ} \mathrm{C}$ in activating buffer $[50 \mathrm{mM}$ Tris- $\mathrm{HCl}(\mathrm{pH} 7.4)$, containing $5 \mathrm{mM}$ $\mathrm{CaCl}_{2}$ and $150 \mathrm{mM} \mathrm{NaCl}$ for gelatinases; $50 \mathrm{mM}$ Tris ( $\mathrm{pH} 7.4$ ), for plasminogen activators (PAs)]. The gels were stained with Coomassie Blue R 250 (Bio-Rad Laboratories, Inc., Hercules, CA, USA) dissolved in a mixture of methanol-acetic acid-water (4:1:5) for $1 \mathrm{~h}$. The gels were then washed in the same solution without dye. Enzyme activity was visualized as distinct bands, indicating proteolysis of the substrate. Images were recorded and band intensities were quantified using the Alliance LD2 system (UVItec, Cambridge, UK).

Quantification of GFs. LR-PGs and LP-PGs samples were assayed for vascular endothelial growth factor (VEGF), thrombospondin-1 (TSP-1), interferon- $\gamma$ (IFN- $\gamma)$, platelet derived growth factor (PDGF)-BB, -AA and -B, tumor growth factor- $\beta 1$ (TGF- $\beta 1)$, tumor necrosis factor- $\alpha$ (TNF- $\alpha$ ) and fibroblast growth factor-7 (FGF-7, also known as keratinocyte growth factor). Commercially available human ELISA kits were used. VEGF-A ELISA kit was purchased from Diaclone (Besançon, France; cat no. 650.080.096). TSP-1 ELISA kit was purchased from Cusabio Biotech Co., Ltd. (College Park, MD, USA; cat no. CSB-E08763 h). IFN- $\gamma$, PDGF-BB, -AA and -B, TGF- $\beta 1$, TNF- $\alpha$ and FGF-7 ELISA kits were purchased from Elabscience (Bethesda, MD, USA; cat nos. E-EL-H0108, E-EL-H1577, E-EL-H1575, E-EL-H1670, E-EL-H10110, E-EL-H0109 and E-EL-H0092). Samples and standards were analyzed in duplicate according to the manufacturer's protocol. The concentration of each GF in the original standard or sample was proportional to the amount of signal produced and was extrapolated from standard curves.

GFs were also measured to test their stability over time and temperature. Freshly obtained supernatants from 2 LR-PG were prepared as described above and divided into aliquots. One aliquot was immediately stored at $-80^{\circ} \mathrm{C}$ to prevent GF degradation, while the others were stored at 4,22 or $37^{\circ} \mathrm{C}$ for 48 or $168 \mathrm{~h}$. As soon as the incubation time expired, the supernatants were stored at $-80^{\circ} \mathrm{C}$. At $37^{\circ} \mathrm{C}$, incubation periods of 24 and $72 \mathrm{~h}$ were also evaluated. Samples were successively analyzed using ELISA kits.
Platelet and white blood cell counts of LR-PGs and LP-PGs samples used for GF quantification are presented in Table I.

Statistical analysis. All data shown are from at least three independent experiments and are expressed as the mean \pm standard deviation. Statistically significant differences between groups were determined using the Student's t-test. Calculations were performed using GraphPad Prism 4 software (GraphPad Software, Inc., La Jolla, CA, USA). P $<0.05$ was considered to indicate a statistically significant difference.

\section{Results}

LR-PGs and LP-PGs affect angiogenesis-related processes. The current study aimed to evaluate whether leukocyte depletion could alter the effectiveness of PG at stimulating angiogenesis-related activities in endothelial cells $(12,13)$. Thus, in vitro experiments were conducted on HUVECs treated with different concentrations of LR-PGs and LP-PGs.

Cells were treated with $1.5 \times 10^{6}$ and $2.5 \times 10^{6}$ platelets $/ \mu 1$ for $72 \mathrm{~h}$. At the lower concentration, both LR-PGs and LP-PGs stimulated proliferation to a greater extent (approximately 2-fold) compared with untreated cells, and no significant differences between LR-PGs and LP-PGs-treated cells were observed. The higher platelet concentration exhibited a weak stimulation in LR-PGs, while LP-PGs appeared to have a slight negative effect compared with untreated cells (Fig. 1A).

To investigate any differences in the ability of LR-PGs and LP-PGs to induce formation of a capillary-like network, a tube formation assay was performed. LR-PGs and LP-PGs $\left(1.5 \times 10^{6}\right.$ platelets $\left./ \mu 1\right)$ both stimulated the formation of more structured tubes compared with untreated cells (Fig. 1B). No significant differences were observed in the effects of LR-PGs and LP-PGs (Fig. 1C).

The effect of LR-PGs and LP-PGs on HUVEC motility was evaluated using a wound-healing assay. At lower concentrations $\left(0.3 \times 10^{6}\right.$ and $1.5 \times 10^{6}$ platelets $\left./ \mu 1\right)$, cells were able to heal the wound to a greater extent compared with the higher concentration $\left(2.5 \times 10^{6}\right.$ platelets $/ \mu$; Fig. 2$)$. With the exception of the highest concentration (at which LP-PGs exhibited a greater negative effect compared with LR-PGs), no significant differences were observed between LR-PGs- and LP-PGs-treated cells.

LR-PGs and LP-PGs affect human fibroblast behavior. The role of PG-derived GFs on human fibroblasts cells has been described previously (14). The present study investigated whether leukocyte depletion could affect some of the cell properties associated with tissue repair, such as proliferative and motile capacities. Fibroblasts were treated with LR-PGs or LP-PGs at concentrations of $1.5 \times 10^{6}$ and $3.0 \times 10^{6}$ platelets $/ \mu 1$ and proliferation was evaluated. Both supernatants stimulated proliferation to a greater extent (approximately 2.5-fold) compared with untreated cells. No significant differences in the proliferative response were observed between LR-PGsand LP-PGs-treated cells (Fig. 3A).

In a wound-healing assay, cells stimulated with lower concentrations $\left(0.3 \times 10^{6}\right.$ and $1.5 \times 10^{6}$ platelets $\left./ \mu 1\right)$ were able to migrate to a greater extent compared with untreated cells. At a higher concentration $\left(2.5 \times 10^{6}\right.$ platelets $\left./ \mu 1\right)$ fibroblast motility 
Table I. Platelets and white blood cell count of samples used for growth factor quantification.

\begin{tabular}{|c|c|c|c|c|}
\hline \multirow[b]{2}{*}{ Sample } & \multicolumn{2}{|c|}{ Leukocyte-rich platelet concentrate } & \multicolumn{2}{|c|}{ Leukocyte-poor platelet concentrate } \\
\hline & Platelets & White blood cells & Platelets & White blood cells \\
\hline 1 & $2.35 \times 10^{6} / \mu 1$ & $8.6 \times 10^{3} / \mu 1$ & $1.99 \times 10^{6} / \mu 1$ & 0 \\
\hline 2 & $3.42 \times 10^{6} / \mu 1$ & $13.2 \times 10^{3} / \mu 1$ & $2.39 \times 10^{6} / \mu 1$ & 0 \\
\hline 3 & $2.09 \times 10^{6} / \mu 1$ & $11.8 \times 10^{3} / \mu 1$ & $1.69 \times 10^{6} / \mu 1$ & 0 \\
\hline 4 & $2.56 \times 10^{6} / \mu 1$ & $10.6 \times 10^{3} / \mu 1$ & $2.74 \times 10^{6} / \mu 1$ & 0 \\
\hline 5 & $1.84 \times 10^{6} / \mu 1$ & $9.8 \times 10^{3} / \mu 1$ & $1.63 \times 10^{6} / \mu 1$ & 0 \\
\hline 6 & $2.41 \times 10^{6} / \mu 1$ & $11.6 \times 10^{3} / \mu 1$ & & \\
\hline 7 & $1.85 \times 10^{6} / \mu 1$ & $8.1 \times 10^{3} / \mu 1$ & & \\
\hline
\end{tabular}

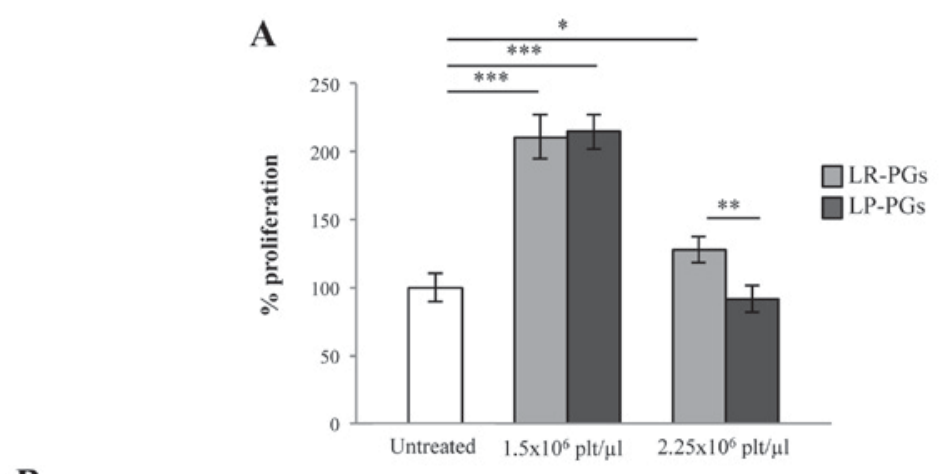

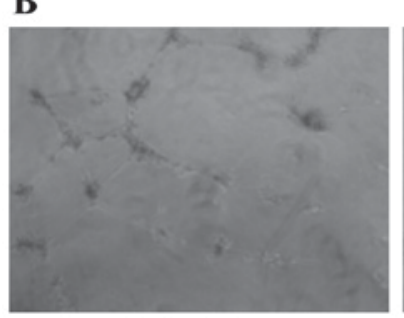

Untreated

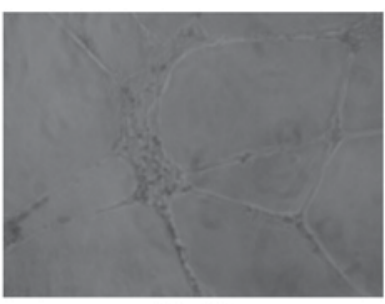

LR-PGs

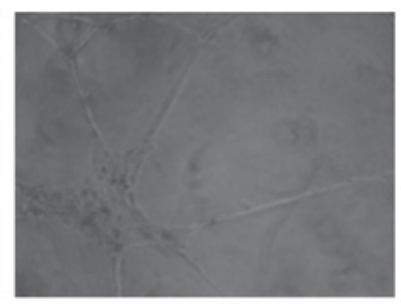

LP-PGs

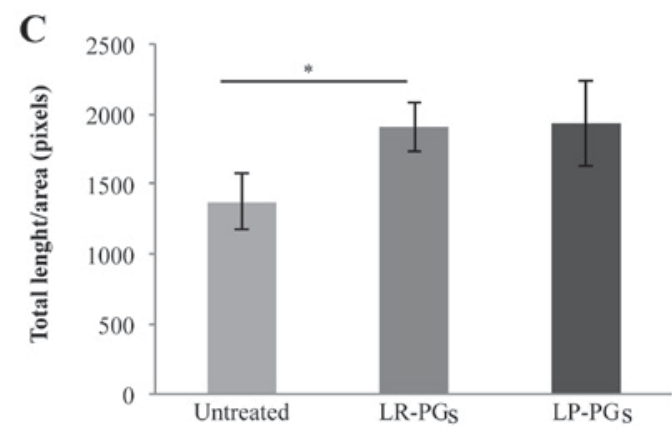

Figure 1. HUVEC proliferation and tube formation. (A) Proliferation of HUVECs treated for $72 \mathrm{~h}$ with LR-PGs and LP-PGs. Data (mean \pm SD of triplicates) represent the \% of proliferation; proliferation of untreated HUVECs was set as $100 \%$. (B) Images shown are representative of tube formation induced by LR-PGs and LP-PGs. (C) Quantification of tubule formation was performed after addition of LR-PGs and LP-PGs at a concentration of $1.5 \times 10^{6}$ platelets $/ \mu 1$. For total length analysis, all images were analyzed with the Angiogenesis Analyzer plugin for ImageJ software. Values derived from 3 independent experiments performed in duplicate were normalized to the same areas of observation field. Data are presented as the mean $\pm \mathrm{SD}$ of total length/field. " $\mathrm{P}<0.05 ;{ }^{* *} \mathrm{P}<0.01$; ${ }^{* * * *} \mathrm{P}<0.001$. LR-PGs, leukocyte-rich platelet gel derived supernatant; LP-PGs, leukocyte-poor platelet gel derived supernatant.

was strongly inhibited (Fig. 3B). No marked differences were evident between LR-PGs- and LP-PGs-treated cells, with the exception of the $1.5 \times 10^{6}$ platelets $/ \mu$ l concentration, where LR-PGs appeared to exert a greater effect on cell motility compared with LP-PGs.
Molecular characterization of LR-PGs and LP-PGs. The activity of gelatinases (MMP-2 and MMP-9) and PAs was assessed using zymography techniques (Fig. 4). Zymography on gelatin substrate identified in both samples (LR-PGs and LP-PGs) the active form of MMP-2 (65 kDa), the pro-enzyme 


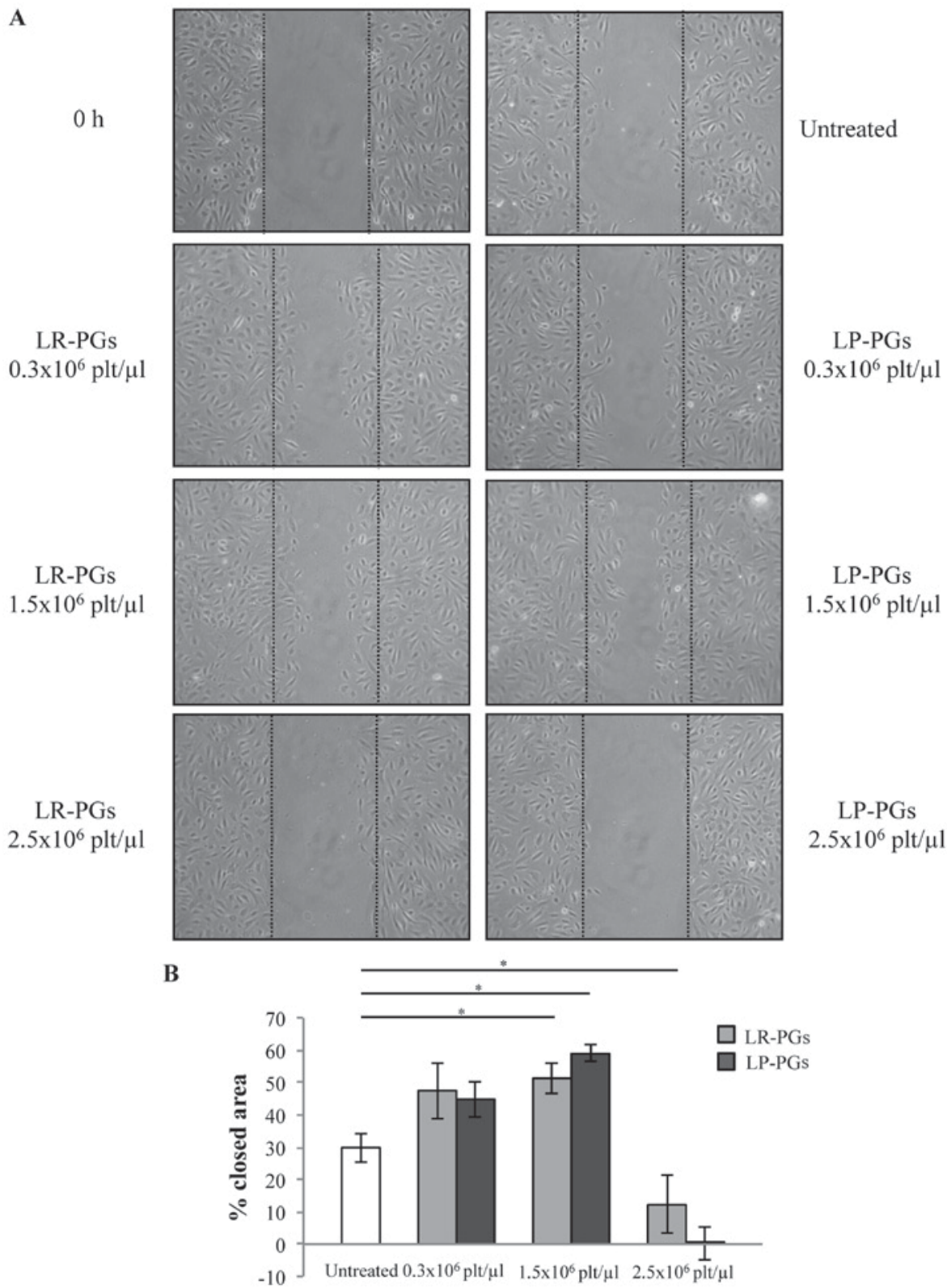

Figure 2. HUVEC motility. LR-PGs and LP-PGs affect HUVEC motility in a wound-healing assay. (A) Representative images recorded $6 \mathrm{~h}$ after wound creation; dotted lines represent the size of the original wound. The upper left image shows the initial status of the wound. (B) Percentage of closed area relative to the original wound area. Data are presented as the mean \pm SD. At $6 \mathrm{~h}$ after wound creation, untreated cells closed $30 \%$ of the wounded area. Cells treated at $0.3 \times 10^{6}$ platelets $/ \mu 1$ closed 47.5 and $44.8 \%$ (with LR-PGs and LP-PGs, respectively). Cells treated at $1.5 \times 10^{6}$ platelets/ $\mu 1$ closed 51.3 and $58.9 \%$ (with LR-PGs and LP-PGs, respectively). Cells treated at the highest concentration $\left(2.5 \times 10^{6}\right.$ platelets $\left./ \mu 1\right)$ closed only $12.2 \%$ when treated with LR-PGs, while no closure was observed in LP-PGs treated cells. "P<0.05. LR-PGs, leukocyte-rich platelet gel derived supernatant; LP-PGs, leukocyte-poor platelet gel derived supernatant.

form of MMP-9 (99 kDa) and two high-molecular-weight bands (128 and $233 \mathrm{kDa}$ ), likely corresponding to gelatinase complexes with their inhibitors (tissue inhibitors of metalloproteinases) (Fig. 4A). No statistically significant differences were evident between the samples (Fig. 4B).

Similarly, zymography of casein-plasminogen substrate showed no significant differences between the two analyzed samples (Fig. 4D). In both samples, the urokinase-type PA (49 kDa) and the complex of PA with its own inhibitor (112 kDa) were present (Fig. 4C).

GF content was analyzed in 5 PGs (PGs 1-5 from Table I) using ELISA kits. The results are presented in Table II. For the majority of GFs, there were no significant differences in GFs content of LR-PGs and LP-PGs. The only exceptions were GP 4 (in which there was a significant decrease in VEGF in LP-PGs compared with LR-PGs), GPs 2 and 5 (in which there was a significant increase in IFN- $\gamma$ in LP-PGs compared with LR-PGs) and GP3 (in which there was a significant increase in PDGF-B in LP-PGs compared with LR-PGs). TNF- $\alpha$ and FGF-7 levels were also measured, but were not detectable.

Effects of time and temperature on GF stability. Two samples (PGs 6 and 7 from Table I) were assayed to verify whether short-term storage at different temperatures could cause degradation of GFs. VEGF, TSP-1, IFN- $\gamma$, PDGF-AA and PDGF-B levels were measured using ELISA kits. The results are presented in Table III. For each sample, no significant 
A

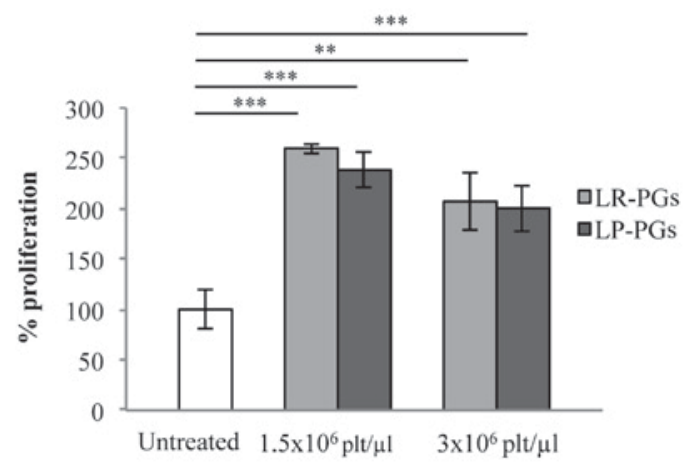

B
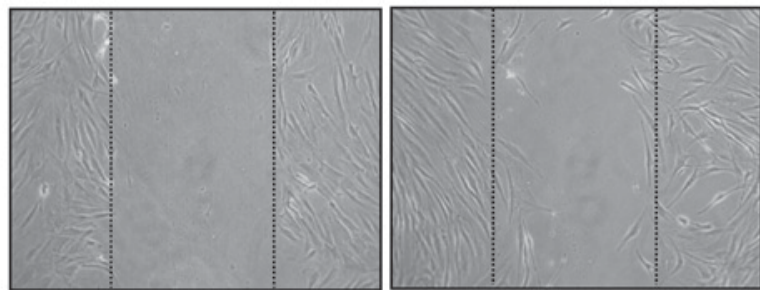

Untreated

$0 \mathrm{~h}$
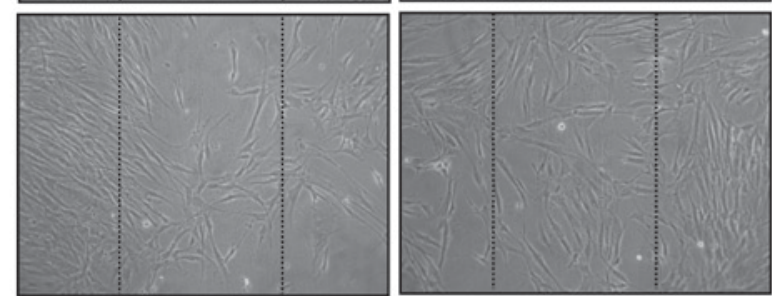

LP-PGs

LR-PGs
$0.3 \times 10^{6} \mathrm{plt} / \mu$
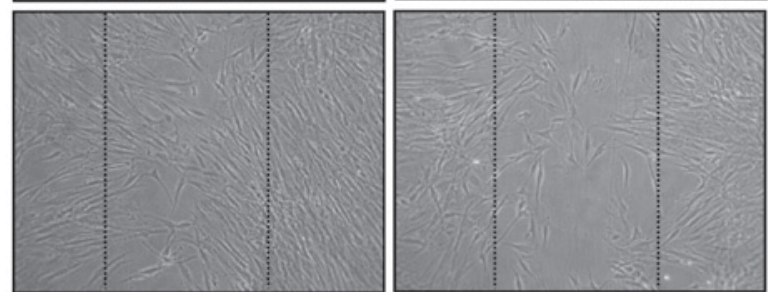

$0.3 \times 10^{6} \mathrm{plt} / \mu \mathrm{l}$

LR-PGs

$1.5 \times 10^{6} \mathrm{plt} / \mu \mathrm{l}$
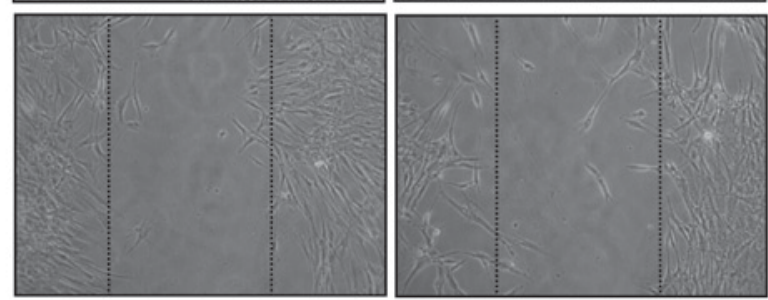

LP-PGs

$1.5 \times 10^{6} \mathrm{plt} / \mu \mathrm{l}$

LP-PGs

$2.5 \times 10^{6} \mathrm{plt} / \mu \mathrm{l}$

Figure 3. NHDF proliferation and motility. (A) LR-PGs and LP-PGs affect NHDF proliferation. Data (mean \pm SD of triplicates) represent the \% of proliferation; proliferation of untreated NHDF was set as $100 \%{ }^{* * *} \mathrm{P}<0.01 ;{ }^{* * * *} \mathrm{P}<0.005$. (B) LR-PGs and LP-PGs affect NHDF motility when tested by (B) wound-healing assay. Representative images shown in the panel were recorded $24 \mathrm{~h}$ after the wound creation; dotted lines represent the size of original wound. The upper left image shows the initial status of the wound. LR-PGs, leukocyte-rich platelet gel derived supernatant; LP-PGs, leukocyte-poor platelet gel derived supernatant.

differences were observed in aliquots maintained for $24-168 \mathrm{~h}$ at 4,22 or $37^{\circ} \mathrm{C}$ compared with the aliquot at point zero.

\section{Discussion}

It is well established that platelet-enriched blood derivatives, such as PRP, contribute to tissue healing, since they carry all the elements involved in this process (platelets, leukocytes and GFs). Establishing the role and importance of each of these factors is essential in order to identify the most effective product to induce tissue healing. As the breadth of knowledge has increased in this field, and the clinical use of PRP has become more common, novel methods of preparation have been developed, offering specific platelet/leukocyte concentrations (15). Different classifications of PCs have been proposed based on their content of leukocytes; LR-PRP and LP-PRP both contain hyperconcentrated platelets but with and without leukocytes, respectively (16-18).

It is still a subject of debate whether leukocytes have a positive or negative effect on healing processes (15). They may have beneficial effects because they stimulate the immune response against infections (19), but it has been recently demonstrated that L-PRP and P-PRP generally inhibit bacterial growth in a comparable way (20); leukocytes also increase GF release, contributing to angiogenesis, matrix production and hypercellularity (21). On the other hand, leukocytes may 
A

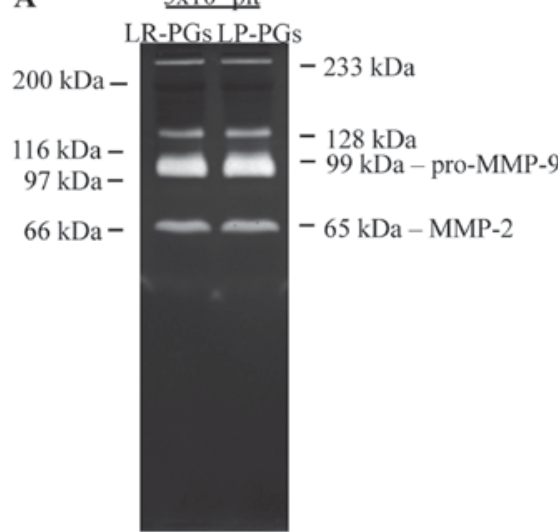

C

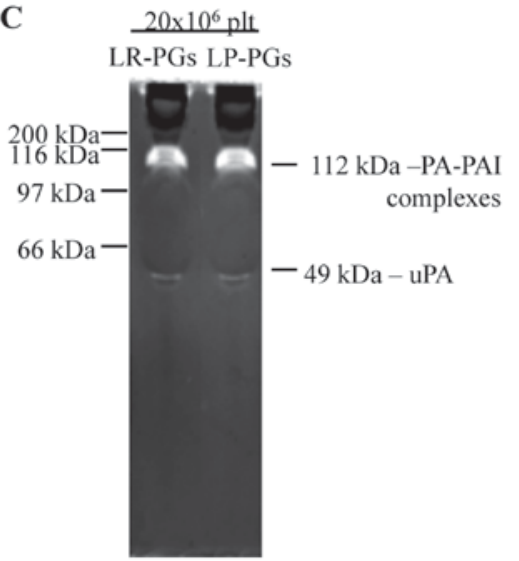

B

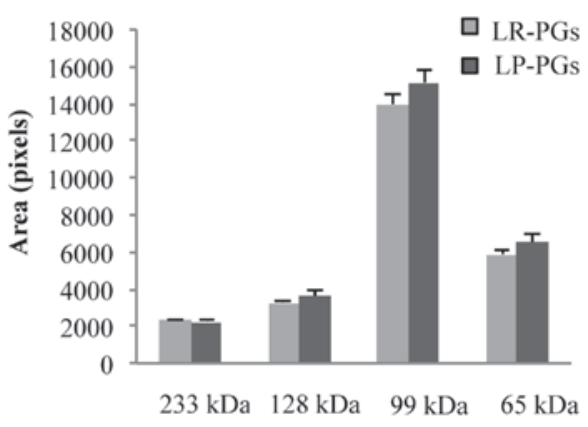

D

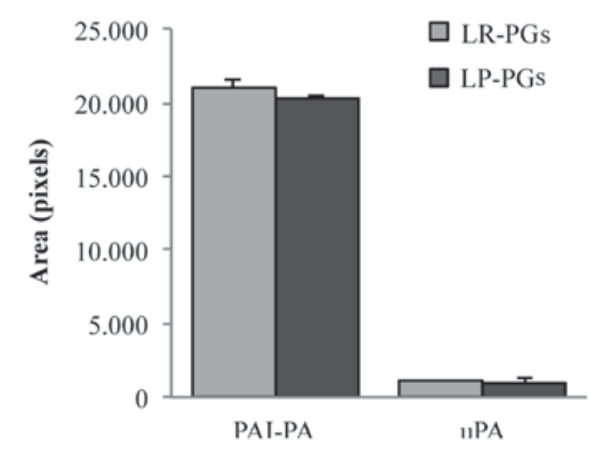

Figure 4. Enzyme content. (A) 5x10 (as highlighted by the different molecular weight lytic bands). (B) Quantification of aforementioned bands indicated no difference in gelatinase activities between LR-PGs and LP-PGs. (C) 20x10 ${ }^{6}$ platelets of LR-PGs and LP-PGs were analyzed by means of casein-plasminogen zymography, showing the presence of uPA and PA-PAI complexes (as highlighted by the different molecular weight lytic bands). (D) Quantification of aforementioned bands indicated no difference in PA activity between LR-PGs and LP-PGs. LR-PGs, leukocyte-rich platelet gel derived supernatant; LP-PGs, leukocyte-poor platelet gel derived supernatant; MMP-9, matrix metalloproteinases-9; MMP-2, matrix metalloproteinases-2; PA, plasminogen activator; PAI, plasminogen activator inhibitor; uPA, plasminogen activator urokinase type.

release inflammatory cytokines (such as TNF- $\alpha$ and reactive oxygen species) that may increase inflammation, having a detrimental effect by delaying tissue healing $(17,22)$. In addition, leukocytes could increase levels of MMPs, which could play an important role in causing matrix degradation and, thus, inferior repair of wounded tissues and scar formation (15); some MMPs, including MMP-9, are stored in circulating platelets and also in neutrophils and are released after their activation (23). Certain studies, mainly conducted in the orthopedic field, have suggested that LP-PRP could induce more effective tissue healing when compared to LR-PRP $(15,24,25)$, while other studies highlight no significant differences between the two $(26,27)$.

It is likely that both LR- and LP-PRP could have positive or negative effects depending on the particular clinical conditions $(2,17)$. In our opinion, there are some aspects still to be examined in relation to the effects of leukocytes contained in PRP on the healing process. In particular, studies outside the areas of tendinopathy and orthopedics are required, in order to investigate effects in a generic wound, where several cell systems are involved. These include endothelial cells, which are responsible for angiogenesis, the formation of new blood vessels that support healing by providing nutrients, promoting granulation tissue formation and facilitating the clearance of debris (28). Fibroblasts are also involved in wound healing, since they play a major role in producing collagen and other matrix components. The current paper, therefore, aimed to provide in vitro data about the positive or negative effects of leukocytes contained in PRP in the healing process, in the context of $\mathrm{PG}$ preparations/formulations.

For this purpose, we obtained PCs from whole blood that were divided into two aliquots, one of which was maintained unaltered (LR-PC), while the other one was leukocyte-depleted by means of filtration (LP-PC). Platelet clotting to obtain LR-PG and LP-PG was induced by calcium and thrombin addition, in order to mimic a 'physiological' activation. During the 30-min period from first clot formation to supernatant recovery, both platelets and leukocytes should be activated; platelet activation and degranulation are simultaneous to clotting, and leukocytes are activated by PDGF released from platelets in a short time period $(29,30)$. The obtained supernatant contains GFs and other biological active molecules released from platelets and leukocytes during activation, which support the tissue repair/regeneration process $(2-4,31)$. This was previously demonstrated to be equivalent, in terms of its in vitro effect, to PG itself (13). Thus, in the present study, for greater ease of use, experiments were performed with supernatants. Since preliminary studies 
Table II. GFs content in LR-PG and LP-PG derived supernatants.

Variables $\quad$ VEGF pg/ml TSP- $1 \mu \mathrm{g} / \mathrm{ml} \quad$ INF $\gamma \mathrm{pg} / \mathrm{ml} \quad$ PDGF-BB pg/ml PDGF-AA pg/ml PDGF-B pg/ml TGF- $\beta 1 \mu \mathrm{g} / \mathrm{ml}$

\begin{tabular}{lcclcrcc}
\hline LR-PG 1 & $1,774.4 \pm 87.8$ & $567.5 \pm 51.3$ & $402.4 \pm 11.4$ & $38.7 \pm 4.5$ & $16.3 \pm 1.4$ & $20.9 \pm 0.8$ & $44.3 \pm 5.7$ \\
LP-PG 1 & $1,954.1 \pm 24.7$ & $576.5 \pm 23.6$ & $510.9 \pm 57.1$ & $29.5 \pm 6.7$ & $15.1 \pm 3.1$ & $289 \pm 4.7$ & $54.2 \pm 4.8$ \\
LR-PG 2 & $686.7 \pm 15.9$ & $342.1 \pm 16.7$ & $241.6 \pm 15.9$ & $40.4 \pm 3.8$ & $33.9 \pm 7.4$ & $59.1 \pm 10.2$ & $36.8 \pm 7.9$ \\
LP-PG 2 & $712.3 \pm 20.5$ & $483.2 \pm 23.8$ & $438.8 \pm 2.2$ & $63.9 \pm 4.7$ & $58.3 \pm 12.1$ & $72.1 \pm 9.1$ & $59.4 \pm 6.9$ \\
LR-PG 3 & $1,166.0 \pm 7.8$ & $559.1 \pm 19.7$ & $547.5 \pm 4.4$ & $125.7 \pm 11.3$ & $57.7 \pm 4.2$ & $80.4 \pm 0.8$ & $64.6 \pm 4.2$ \\
LP-PG 3 & $1,080.1 \pm 51.3$ & $501.3 \pm 18.7$ & $653.6 \pm 35.0$ & $99.4 \pm 8.7$ & $60.6 \pm 0.8$ & $93.6 \pm 3.6$ & $88.8 \pm 9.8$ \\
LR-PG 4 & $1,423.9 \pm 10.6$ & $487.3 \pm 39.6$ & $362.1 \pm 11.3$ & $117.7 \pm 9.7$ & $54.9 \pm 1.1$ & $101.6 \pm 9.4$ & $58.9 \pm 4.7$ \\
LP-PG 4 & $1,261.5 \pm 31.8$ & $474.9 \pm 41.3$ & $353.6 \pm 13.5$ & $144.3 \pm 12.1$ & $63.3 \pm 7.9$ & $80.7 \pm 2.3$ & $48.7 \pm 6.9$ \\
LR-PG 5 & $2,663.3 \pm 60.1$ & $421.9 \pm 54.8$ & $282.9 \pm 10.7$ & $162.9 \pm 8.2$ & $72 \pm 5.9$ & $86.4 \pm 5.3$ & $43.8 \pm 11.3$ \\
LP-PG 5 & $2,464.8 \pm 73.6$ & $546.7 \pm 51.3$ & $471.5 \pm 6.0$ & $141.6 \pm 9.2$ & $76.2 \pm 5.2$ & $97.8 \pm 32.9$ & $73.0 \pm 8.7$
\end{tabular}

GFs content is reported as concentration \pm standard deviation. To take due account to different platelets (plt) $/ \mu 1$ starting count, the concentrations of growth factors and cytokines tested in the examined samples are reported after correction based on their plt $/ \mu 1 \mathrm{concentrations}$ (concentrations reported in table refer to $1 \times 10^{6} \mathrm{plt} / \mu \mathrm{l}$ ). GF, growth factor; VEGF, vascular endothelial growth factor; TSP-1, thrombospondin-1; INF- $\gamma$, interferon- $\gamma$; PDGF-BB, platelet derived growth factor-BB; PDGF-AA, platelet derived growth factor-AA; PDGF-B, platelet derived growth factor-B; TGF- $\beta 1$, tumor growth factor- $\beta 1$.

Table III. Time and temperature effects on GFs contained in LR-PG derived supernatants.

\begin{tabular}{|c|c|c|c|c|c|}
\hline Variables & VEGF pg/ml & $\mathrm{TSP}-1 \mu \mathrm{g} / \mathrm{ml}$ & INF $\gamma \mathrm{pg} / \mathrm{ml}$ & PDGF-AA pg/ml & PDGF-B pg/ml \\
\hline PG 6 point zero & $2,217.0 \pm 94.9$ & $196.7 \pm 17.4$ & $918.0 \pm 41.8$ & $49.1 \pm 11.1$ & $30.5 \pm 2.1$ \\
\hline PG $648 \mathrm{~h} 4^{\circ}$ & $2,122.7 \pm 24.9$ & $152.3 \pm 9.6$ & $913.3 \pm 20.6$ & $44.1 \pm 11.4$ & $35.7 \pm 2.7$ \\
\hline PG 61 week $4^{\circ}$ & $1,927.9 \pm 11.3$ & $148.2 \pm 9.6$ & $936.9 \pm 5.6$ & $52.5 \pm 3.1$ & $31.2 \pm 3$ \\
\hline PG 648 h $22^{\circ}$ & $2,130.7 \pm 31.2$ & $169.8 \pm 11.8$ & $834.6 \pm 108.9$ & $43.5 \pm 8.9$ & $36.5 \pm 2.6$ \\
\hline PG 61 week $22^{\circ}$ & $2,022.1 \pm 58.7$ & $244.8 \pm 17.5$ & $932.5 \pm 31.9$ & $57.3 \pm 7.5$ & $28.7 \pm 4.2$ \\
\hline PG 624 h $37^{\circ}$ & $2,119.5 \pm 15.8$ & $161.1 \pm 10.3$ & $902.0 \pm 28$ & $43.4 \pm 9.2$ & $27.9 \pm 3$ \\
\hline PG 648 h $37^{\circ}$ & $2,202.6 \pm 169.4$ & $160.4 \pm 9.5$ & $949.1 \pm 8.4$ & $39.7 \pm 4.9$ & $24.9 \pm 3.7$ \\
\hline PG $672 \mathrm{~h} 37^{\circ}$ & $2,453.4 \pm 207.8$ & $157.2 \pm 13.6$ & $900.2 \pm 77.5$ & $41.6 \pm 9.2$ & $35.1 \pm 2.1$ \\
\hline PG 61 week $37^{\circ}$ & $2,547.6 \pm 24.8$ & $146.2 \pm 13.9$ & $794.6 \pm 21.2$ & $41.9 \pm 9.5$ & $37.8 \pm 5.2$ \\
\hline PG 7 point zero & $656.3 \pm 44.2$ & $206.4 \pm 12.9$ & $1,195.7 \pm 13.6$ & $27.2 \pm 4.9$ & $22.1 \pm 5.4$ \\
\hline PG $748 \mathrm{~h} 4^{\circ}$ & $539.6 \pm 56$ & $198.2 \pm 18.8$ & $1,189.1 \pm 51.9$ & $26.2 \pm 3.2$ & $21.4 \pm 4.7$ \\
\hline PG 71 week $4^{\circ}$ & $541.7 \pm 23.6$ & $214.2 \pm 11.8$ & $1,243.7 \pm 2.9$ & $25.1 \pm 2.4$ & $40.1 \pm 8.1$ \\
\hline PG 748 h $22^{\circ}$ & $591.7 \pm 5.9$ & $213.2 \pm 11.4$ & $1,206.6 \pm 28.3$ & $26.5 \pm 2.2$ & $24 \pm 4.1$ \\
\hline PG 71 week $22^{\circ}$ & $606.3 \pm 14.7$ & $210.7 \pm 5.7$ & $1,245.7 \pm 25.9$ & $24.3 \pm 0.9$ & $22.2 \pm 3.2$ \\
\hline PG 724 h $37^{\circ}$ & $658.4 \pm 41.3$ & $164.7 \pm 14.3$ & $1,245.7 \pm 23.6$ & $26.9 \pm 0.4$ & $22 \pm 2.1$ \\
\hline PG 748 h $37^{\circ}$ & $623.0 \pm 26.5$ & $177.4 \pm 12.1$ & $1,220.3 \pm 24.7$ & $28.3 \pm 3.5$ & $37.1 \pm 9.6$ \\
\hline PG 772 h $37^{\circ}$ & $785.5 \pm 55.9$ & $170.2 \pm 15.9$ & $1,245.73 \pm 25.6$ & $45.0 \pm 24.1$ & $21.8 \pm 1.4$ \\
\hline PG 71 week $37^{\circ}$ & $887.6 \pm 70.7$ & $224.0 \pm 8.6$ & $1,221.4 \pm 34.5$ & $24.6 \pm 1.6$ & $23 \pm 1.2$ \\
\hline
\end{tabular}

GFs content is reported as concentration \pm standard deviation. To take due account to different platelets $/ \mu 1$ starting count, the concentrations of growth factors and cytokines tested in the examined samples is reported after being corrected based on their platelet/ $\mu 1$ concentrations (concentrations reported in table refer to $1 \times 10^{6} \mathrm{plt} / \mu 1$ ). VEGF, vascular endothelial growth factor; TSP-1, thrombospondin- 1 ; INF- $\gamma$, interferon- $\gamma$; PDGF-AA, platelet derived growth factor-AA; PDGF-B, platelet derived growth factor-B.

already highlighted that different concentrations (expressed as platelets $/ \mu 1)$ exert different biological effects $(12-14,32)$, specific concentrations were examined. Finally, since the concentration of biologically relevant molecules released from platelets is proportional to the initial concentration of platelets $/ \mu 1$, we assumed that the supernatant would maintain the same platelets/ $\mu$ l concentration of PG from which it was obtained, even if the platelets were no longer physically present.

HUVECs were tested for the characteristics required for the angiogenesis process, such as proliferation and motility. As it was already known that some concentrations are more effective than others (13), cells were treated with $1.5 \times 10^{6}$ platelets $/ \mu 1$ and $2.5 \times 10^{6}$ platelets $/ \mu 1$ (optimal and counterproductive 
concentrations for endothelial cells activity, respectively). LR-PGs and LP-PGs both stimulated proliferation and motility, but no significant differences between LR-PGs- and LP-PGs-treated cells were observed. The tube formation assay, which was only conducted at the optimal concentration for endothelial cells, indicated the same trend.

Human fibroblasts were also evaluated for proliferation and motility in response to LR-PGs and LP-PGs. The results were consistent with those obtained for endothelial cells. In the wound-healing assay, unlike HUVEC cells (that move into the wound as a compact layer) fibroblasts migrate into the wounded space individually. This made it impossible to perform an accurate quantification of the closed area; however, upon simple observation of the images, it is evident that at lower concentrations, cells are able to migrate into the wound to a greater extent compared with untreated cells, whereas motility was strongly inhibited at the highest concentration. No notable differences, however, were observed between LR-PGs- and LP-PGs-treated cells.

In conclusion, it seems that LR-PGs and LP-PGs are equally effective in inducing biological activities relevant for tissue healing in endothelial cells and fibroblasts if used at optimal concentrations. At higher, detrimental concentrations, the LP-PGs exerts a slight negative effect compared with LR-PGs.

Enzyme levels were measured in order to assess whether LR-PGs and LP-PGs contribute differently in terms of proteolytic enzyme activity during tissue repair. White blood cells release MMPs and PAs, which are able to remodel the extracellular matrix (33-35); however, if excessive amounts are released, this could counteract tissue healing (17). Zymographies showed similar levels of expression of both gelatinases and PAs, suggesting that leukocyte depletion does not affect these enzyme activities.

In order to understand whether leukocyte removal impoverishes the PG in terms of GFs that promote healing processes, it was critical to assess GFs in LR-PGs and LP-PGs. Some studies on GFs in PRP have already been conducted, but these are not relevant if quantification was performed on non-activated platelet suspension; in order to properly measure GF content, the PRP needs to be activated in PG $(2,21)$. In the present study, several GFs were assayed to measure their levels in LR-PGs and LP-PGs. The GFs we selected are involved in various aspects of wound repair: VEGF has a leading role in angiogenesis (36); TSP-1 has a well-defined role in latent TGF- $\beta 1$ activation and in inflammation (37,38); IFN- $\gamma$ can delay the wound healing process (39); PDGFs are involved in tissue remodeling (40); TGF- $\beta$ affects inflammation, angiogenesis and fibroblast activities (41); TNF- $\alpha$ stimulates fibroblast proliferation and angiogenesis (42); and FGF-7 stimulates proliferation, migration and angiogenesis (42). When LR-PGs and LP-PGs were compared for each GF, there were few differences, generally supporting the proposal that no differences are induced by the leukocyte depletion process. A small number of GFs showed significant differences: In GP 4, the VEGF level decreased in the LP-PGs compared with LR-PGs, while in GPs 2 and 5, the IFN- $\gamma$ level increased in LP-PGs compared with LR-PGs. We hypothesized that the eventual lower content of VEGF (which positively drives angiogenesis) and higher content of IFN- $\gamma$ (which negatively affects wound healing) in LP-PGs could partially explain the more negative effect induced by LP-PGs in the proliferation and motility tests.

The present data confirmed the ability of PG to stimulate the biological mechanisms involved in the repair/regeneration of wounds. The data also revealed a substantial overlap of performance between LR-PG and LP-PG, which could probably be explained by a substantial equivalence in GFs and enzyme content. GFs and enzymes are not negatively affected by leukocyte depletion and are still present in sufficient amounts to sustain in vitro repair of endothelial cell and fibroblast cultures.

Finally, in a preliminary way, some GFs were also analyzed in two samples to verify how short-term storage at various temperatures could affect GF stability. The results did not support the hypothesis that preservation for short periods ( $24 \mathrm{~h}$ to 1 week) at easily obtainable temperatures [such as refrigerator temperature $\left(\sim 4^{\circ} \mathrm{C}\right)$ or room temperature $\left(\sim 22^{\circ} \mathrm{C}\right)$ ] can influence the content in GFs. Even maintaining the samples at body temperature $\left(37^{\circ} \mathrm{C}\right)$ for $24 \mathrm{~h}$ to 1 week did not significantly affect the stability of the GFs, suggesting that they remained fully active during the entire duration of clinical treatments. However, assays with a larger number of samples are required to confirm these findings.

Although the present study was limited by the absence of in vivo experiments, we hypothesize that, in in vivo applications, a priori removal of leukocytes is not a fundamental requirement to obtain greater efficacy of PG. Furthermore, each clinical situation will require careful consideration. Data from the analyses on GF stability also suggest it may be possible to design PG formulations that can be safely stored by patients in a domestic setting and autonomously applied (for example, eye drops for ophthalmologic treatments) (9). This would avoid the requirement for daily medical attention.

\section{Acknowledgements}

This study was partially supported by Fondazione Cassa di Risparmio della Provincia dell'Aquila.

\section{References}

1. Marx RE: Platelet-rich plasma (PRP): What is PRP and what is not PRP? Implants Dent 10: 225-228, 2001.

2. Arnoczky SP, Delos D and Rodeo SA: What is platelet-rich plasma? Oper Tech Sports Med 19: 142-148, 2011.

3. Blair P and Flaumenhaft R: Platelet alpha-granules: Basic biology and clinical correlates. Blood Rev 23: 177-189, 2009.

4. Leslie M: Cell biology. Beyond clotting: The powers of platelets. Science 328: 562-564, 2010.

5. Knezevic NN, Candido KD, Desai R and Kaye AD: Is platelet-rich plasma a future therapy in pain management? Med Clin North Am 100: 199-217, 2016.

6. Lacci KM and Dardik A: Platelet-rich plasma: Support for its use in wound healing. Yale J Biol Med 83: 1-9, 2010.

7. Mihaylova Z, Mitev V, Stanimirov P, Isaeva A, Gateva N and Ishkitiev $\mathrm{N}$ : Use of platelet concentrates in oral and maxillofacial surgery: An overview. Acta Odontol Scand 75: $1-11,2017$.

8. Fréchette JP, Martineau I and Gagnon G: Platelet-rich plasmas: Growth factor content and roles in wound healing. J Dent Res 84: 434-439, 2005

9. van der Meer PF, Seghatchian J and Marks DC: Quality standards, safety and efficacy of blood-derived serum eye drops: A review. Transfus Apher Sci 54: 164-167, 2016.

10. Hsu WK, Mishra A, Rodeo SR, Fu F, Terry MA, Randelli P, Canale ST and Kelly FB: Platelet-rich plasma in orthopaedic applications: Evidence-based recommendations for treatment. J Am Acad Orthop Surg 21: 739-748, 2013. 
11. Lee KS: Platelet-rich plasma injection. Semin Musculoskelet Radiol 17: 91-98, 2013.

12. Rughetti A, Giusti I, D'Ascenzo S, Leocata P, Carta G, Pavan A, Dell'Orso L and Dolo V: Platelet gel-released supernatant modulates the angiogenic capability of human endothelial cells. Blood Transfus 6: 12-17, 2008.

13. Giusti I, Rughetti A, D'Ascenzo S, Millimaggi D, Pavan A, Dell'Orso L and Dolo V: Identification of an optimal concentration of platelet gel for promoting angiogenesis in human endothelial cells. Transfusion 49: 771-778, 2009.

14. Giusti I, Rughetti A, D'Ascenzo S, Di Stefano G, Nanni MR, Millimaggi D, Dell'orso L and Dolo V: The effects of platelet gel-released supernatant on human fibroblasts. Wound Repair Regen 21: 300-308, 2013.

15. Cross JA, Cole BJ, Spatny KP, Sundman E, Romeo AA, Nicholson GP, Wagner B and Fortier LA: Leukocyte-reduced platelet-rich plasma normalizes matrix metabolism in torn human rotator cuff tendons. Am J Sports Med 43: 2898-2906, 2015.

16. Dohan Ehrenfest DM, Bielecki T, Mishra A, Borzini P, Inchingolo F, Sammartino G, Rasmusson L and Evert PA: In search of a consensus terminology in the field of platelet concentrates for surgical use: Platelet-rich plasma (PRP), platelet-rich fibrin (PRF), fibrin gel polymerization and leukocytes. Curr Pharm Biotechnol 13: 1131-1137, 2012.

17. Zhou Y, Zhang J, Wu H, Hogan MV and Wang JH: The differential effects of leukocyte-containing and pure platelet-rich plasma (PRP) on tendon stem/progenitor cells-implications of PRP application for the clinical treatment of tendon injuries. Stem Cell Res Ther 6: 173, 2015.

18. Duif C, Vogel T, Topcuoglu F, Spyrou G, von Schulze Pellengahr C and Lahner M: Does intraoperative application of leukocyte-poor platelet-rich plasma during arthroscopy for knee degeneration affect postoperative pain, function and quality of life? A 12-month randomized controlled double-blind trial. Arch Orthop Trauma Surg 135: 971-977, 2015.

19. Moojen DJ, Everts PA, Schure RM, Overdevest EP, van Zundert A, Knape JT, Castelein RM, Creemers LB and Dhert WJ: Antimicrobial activity of platelet-leukocyte gel against Staphylococcus aureus. J Orthop Res 26: 404-410, 2008.

20. Mariani E, Canella V, Berlingeri A, Bielli A, Cattini L, Landini MP, Kon E, Marcacci M, Di Matteo B and Filardo G: Leukocyte presence does not increase microbicidal activity of Platelet-rich Plasma in vitro. BMC Microbiol 15: 149, 2015.

21. Dohan Ehrenfest DM, Bielecki T, Jimbo R, Barbé G, Del Corso M, Inchingolo F and Sammartino G: Do the fibrin architecture and leukocyte content influence the growth factor release of platelet concentrates? An evidence-based answer comparing a pure platelet-rich plasma (P-PRP) gel and a leukocyte- and platelet-rich fibrin (L-PRF). Curr Pharm Biotechnol 13: 1145-1152, 2012.

22. Dragoo JL, Braun HJ, Durham JL, Ridley BA, Odegaard JI, Luong R and Arnoczky SP: Comparison of the acute inflammatory response of two commercial platelet-rich plasma systems in healthy rabbit tendons. Am J Sports Med 40: 1274-1281, 2012.

23. Hasty KA, Pourmotabbed TF, Goldberg GI, Thompson JP, Spinella DG, Stevens RM and Mainardi CL: Human neutrophil collagenase: A distinct gene product with homology to other matrix metalloproteinases. J Biol Chem 265: 11421-11424, 1990.

24. McCarrel TM, Minas T and Fortier LA: Optimization of leukocyte concentration in platelet rich plasma for the treatment of tendinopathy. J Bone Joint Surg Am 94: e143(1-8), 2012.

25. Yin WJ, Xu HT, Sheng JG, An ZQ, Guo SC, Xie XT and Zhang CQ: Advantages of pure platelet-rich plasma compared with leukocyte- and platelet-rich plasma in treating rabbit knee osteoarthritis. Med Sci Monit 22: 1280-1290, 2016.
26. Filardo G, Kon E, Pereira Ruiz MT, Vaccaro F, Guitaldi R, Di Martino A, Cenacchi A, Fornasari PM and Marcacci M: Platelet-rich plasma intra-articular injections for cartilage degeneration and osteoarthritis: Single-vs. double-spinning approach. Knee Surg Sports Traumatol Arthrosc 20: 2082-2091, 2012.

27. Riboh JC, Saltzman BM, Yanke AB, Fortier L and Cole BJ: Effect of leukocyte concentration on the efficacy of platelet rich plasma in the treatment of knee osteoarthritis. Am J Sports Med 44: 792-800, 2016.

28. Diegelmann RF and Evans MC: Wound healing: An overview of acute, fibrotic and delayed healing. Front Biosci 9: 283-289, 2004.

29. Tzeng DY, Deuel TF, Huang JS and Baehner RL: Platelet-derived growth factor promotes human peripheral monocyte activation. Blood 66: 179-183, 1985.

30. Elstad MR, McIntyre TM, Prescott SM and Zimmerman GA: The interaction of leukocytes with platelets in blood coagulation. Curr Opin Hematol 2: 47-54, 1995.

31. Grazul-Bilska AT, Johnson ML, Bilski JJ, Redmer DA, Reynolds LP, Abdullah A and Abdullah KM: Wound healing: The role of growth factors. Drugs Today (Barc) 39: 787-800, 2003.

32. Giusti I, D'Ascenzo S, Mancò A, Di Stefano G, Di Francesco M, Rughetti A, Dal Mas A, Properzi G, Calvisi V and Dolo V: Platelet concentration in platelet-rich plasma affects tenocyte behavior in vitro. Biomed Res Int 2014: 630870, 2014.

33. Granelli-Piperno A, Vassalli JD and Reich E: Secretion of plasminogen activator by human polymorphonuclear leukocytes. Modulation by glucocorticoids and other effectors. J Exp Med 146: 1693-1706, 1977.

34. Salamonsen LA and Lathbury LJ: Endometrial leukocytes and menstruation. Hum Reprod Update 6: 16-27, 2000.

35. Xue M, Thompson PJ, Clifton-Bligh R, Fulcher G, Gallery ED and Jackson C: Leukocyte matrix metalloproteinase-9 is elevated and contributes to lymphocyte activation in type I diabetes. Int J Biochem Cell Biol 37: 2406-2416, 2005.

36. Hoeben A, Landuyt B, Highley MS, Wildiers H, Van Oosterom AT and DeBruijn EA: Vascular endothelial growth factor and angiogenesis. Pharmacol Rev 56: 549-580, 2004.

37. Soto-Pantoja DR, Shih HB, Maxhimer JB, Cook KL, Ghosh A, Isenberg JS and Roberts DD: Thrombospondin-1 and CD47 signaling regulate healing of thermal injury in mice. Matrix Biol 37: 25-34, 2014

38. Blanco-Mezquita JT, Hutcheon AE and Zieske JD: Role of thrombospondin-1 in repair of penetrating corneal wounds. Invest Ophthalmol Vis Sci 54: 6262-6268, 2013.

39. Laato M, Heino J, Gerdin B, Kähäri VM and Niinikoski J: Interferon-gamma-induced inhibition of wound healing in vivo and in vitro. Ann Chir Gynaecol 90 (Suppl 205): S19-S23, 2001.

40. Martínez CE, Smith PC and Palma Alvarado VA: The influence of platelet-derived products on angiogenesis and tissue repair: A concise update. Front Physiol 6: 290, 2015.

41. Penn JW, Grobbelaar AO and Rolfe KJ: The role of the TGF- $\beta$ family in wound healing, burns and scarring: A review. Int J Burns Trauma 2: 18-28, 2012.

42. Rozman P and Bolta Z: Use of platelet growth factors in treating wounds and soft-tissue injuries. Acta Dermatovenerol Alp Pannonica Adriat 16: 156-165, 2007. 